\title{
ON THE DECAY OF THE ELEMENTS OF INVERSE TRIANGULAR TOEPLITZ MATRICES*
}

\author{
NEVILLE J. FORD ${ }^{\dagger}$, DMITRY V. SAVOSTYANOV ${ }^{\ddagger}$, AND NICKOLAI L. ZAMARASHKIN $§$
}

\begin{abstract}
We consider half-infinite triangular Toeplitz matrices with slow decay of the elements and prove under a monotonicity condition that the elements of the inverse matrix, as well as the elements of the fundamental matrix, decay to zero. We provide a quantitative description of the decay of the fundamental matrix in terms of $p$-norms. The results add to the classical results of Jaffard and Vecchio and are illustrated by numerical examples.
\end{abstract}

Key words. triangular Toeplitz matrix, Volterra equation, Abel kernel, slow decay, stability

AMS subject classifications. 15A09, 15B05, 45E10

DOI. $10.1137 / 130931734$

1. Introduction. Consider a real half-infinite triangular Toeplitz matrix, defined by a sequence $a=\left\{a_{k}\right\}_{k=0}^{\infty}$ as follows:

$$
A=\left[\begin{array}{ccccc}
a_{0} & & & & \\
a_{1} & a_{0} & & & \\
a_{2} & a_{1} & a_{0} & & \\
a_{3} & a_{2} & a_{1} & a_{0} & \\
\cdot & \cdot & \cdot & \cdot & \cdot
\end{array}\right] .
$$

If $a_{0} \neq 0$, the matrix $A$ is invertible, and the inverse matrix $B=A^{-1}$ is also triangular Toeplitz with elements $b=\left\{b_{k}\right\}_{k=0}^{\infty}$ given by the following formula:

$$
b_{0}=\frac{1}{a_{0}}, \quad b_{k}=-\frac{1}{a_{0}} \sum_{j=0}^{k-1} a_{k-j} b_{j} \quad \text { for } \quad k \geq 1 .
$$

Since $A$ and $B$ are triangular, the inverse of the $k \times k$ leading submatrix of $A$ is the $k \times k$ leading submatrix of $B$. Given the asymptotic behavior of $a=\left\{a_{k}\right\}$, what can we say about the asymptotic properties of $b=\left\{b_{k}\right\}$ ?

This study is motivated by the convolution Volterra equation of the first kind,

$$
\int_{0}^{t} K(t-s) f(s) d s=g(t), \quad 0 \leq t \leq T
$$

that describes linear time-invariant causal systems [17, Chapter 2]. The analysis of integral equations of the first kind is generally more difficult than that of the equations

${ }^{*}$ Received by the editors August 5, 2013; accepted for publication (in revised form) by J. G. Nagy July 7, 2014; published electronically October 28, 2014. This work was supported by RSCF grant 1411-00806 at the Institute of Numerical Mathematics RAS during the preparation of the manuscript. http://www.siam.org/journals/simax/35-4/93173.html

$\dagger$ University of Chester, Chester CH1 4BJ, UK (njford@chester.ac.uk).

$\ddagger$ Institute of Numerical Mathematics of Russian Academy of Sciences, Moscow 119333, Russia, and University of Chester, Chester CH1 4BJ, UK (dmitry.savostyanov@gmail.com). This work was started when this author was a visiting research fellow at the University of Chester, supported by the Leverhulme Trust.

$\S$ Institute of Numerical Mathematics of Russian Academy of Sciences, Moscow 119333, Russia (nikolai.zamarashkin@gmail.com). 
of the second kind $[19,3,4,7]$, particularly when the kernel $K(t-s)$ is unbounded at $t=s$. An important example is the Abel kernel $K(t)=t^{-\alpha}, 0<\alpha<1$, and the generalized Abel kernel, which has the same singularity. Equations of Abel type arise for cylindrically symmetrical plasma $[22,20,24]$ and in fractional calculus, which is found in mathematical models in mechanics, biochemistry, electrical engineering, and medicine $[11,8,12]$.

The discretization of the convolution Volterra equation with the generalized Abel kernel on a uniform grid gives a linear system with triangular Toeplitz matrix (1.1). The matrix $A$ inherits certain properties of the kernel $K(t)$, i.e., some or all from the following list:

(a) the elements $a_{k}$ are nonnegative and decay to zero;

(b) the sequence $a_{k}$ is nonincreasing and for large $k$ behaves as $a_{k} \sim(k+1)^{-\alpha}$;

(c) the sequence $a_{k}$ is log-convex, i.e., $a_{k}^{2} \leq a_{k-1} a_{k+1}$ for $k \geq 1$.

Using these properties of $\left\{a_{k}\right\}$, we describe the asymptotic behavior of the elements $\left\{b_{k}\right\}$, e.g., whether the norm of the inverse matrix $B=A^{-1}$ is bounded, which is essential for error analysis; see, e.g., [17].

Log-convexity is arguably the most popular tool for the analysis, applied to both functions [13] and linear systems [17, Chapter 10]. However, it is also a very delicate property that can be lost after discretization, unless special measures are taken to inherit it from the kernel. A notable example is a collocation method on the uniform grid $t_{k}=k h$ with one collocation point $t_{k+c}=(k+c) h$ per interval $\left[t_{k}, t_{k+1}\right]$, applied to the Volterra equation (1.3) with the Abel kernel $K(t)=t^{-\alpha}$. If $0<\alpha<1$ and $0<$ $c \leq 1$, the matrix elements generated by the collocation will be log-convex only for $c>$ $c_{\star}(\alpha)$. The critical value $c_{\star}(\alpha)$ is estimated in [6] and then with improved technique in [18]. However, a slight modification of the kernel $K(t)$ or tiny perturbation of the matrix $A$ may result in the loss of log-convexity, as we demonstrate in section 2.

An effort has been made by several authors to characterize the asymptotic behavior of $\left\{b_{k}\right\}$ based only on the decay properties (a) and (b) of the matrix $A$. From an asymptotic point of view, the following three cases can be considered:

1. fast decay, $\sum_{k=0}^{\infty}\left|a_{k}\right|<\infty$;

2. slow decay, $a_{k} \rightarrow 0, \sum_{k=0}^{\infty}\left|a_{k}\right|=\infty$;

3. stagnation, $a_{k} \rightarrow a_{*}>0$.

The first case includes matrices with superlinear decay, i.e., $\left|a_{k}\right| \leq C(1+k)^{-\alpha}$ for some $\alpha>1$ and $C>1$. They were considered by Jaffard [15] in a very general framework of matrices with Toeplitz-type spatial decay (similar results for band matrices have been shown in [10] and generalized in [1]). The classical result of Jaffard shows that if the inverse matrix $B=A^{-1}$ is bounded, then it has the same polynomial decay of coefficients as $A$. This excludes the situation when the elements of $A$ decay fast, but $B=A^{-1}$ is not bounded, e.g.,

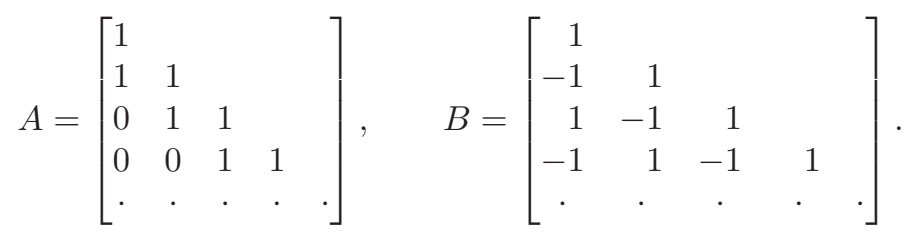

The third case $a_{k} \rightarrow a_{*}>0$ was considered under the monotonicity condition $a_{0} \geq a_{1} \geq a_{2} \geq \cdots$ by Vecchio. An upper bound for the series $\sum_{k=0}^{\infty}\left|b_{k}\right|$ was established in [26] and improved later in [2]. It follows that $b_{k} \rightarrow 0$ and the inverse matrix $B=A^{-1}$ belongs to the first class (with fast decay of the elements). 
Relatively little is known about the second case - the slow decay of the matrix elements. The results of Jaffard do not cover this case. Vecchio mentioned in [26] that partial sums $u_{k}=\sum_{j=0}^{k} b_{j}$ cannot form a converging series. The authors of [2] provide an upper bound for $\sum_{j=0}^{k}\left|b_{j}\right|$ that grows linearly with $k$. However, these results do not say much about the properties of $\left\{b_{k}\right\}$ in the limit.

In this paper we consider matrices $A$ with nonnegative elements $a_{k} \geq 0$ and slow decay $\sum_{k=0}^{\infty} a_{k}=\infty$, assuming without loss of generality (w.l.o.g.) that $a_{0}=1$, and provide new results on $\left\{b_{k}\right\}$ to fill the gap in the existing literature. In section 2 we recall the properties of matrices with log-convex decay. In section 3 under the condition $a_{k-1} \geq a_{k}, k \geq 1$, we prove that $u_{k} \rightarrow 0$ and therefore $b_{k} \rightarrow 0$. In section 4 we describe the decay of $u_{k}$ qualitatively in terms of $p$-norms. In section 5 we present numerical examples, illustrating the theoretical results obtained in this paper.

2. Log-convex case. Following [16], we call a function $f(x) \log$-convex (or superconvex) if $\log f(x)$ is convex. A similar notion is defined for sequences as follows.

Definition 2.1. A sequence $a=\left\{a_{k}\right\}_{k=0}^{\infty}$ is called log-convex if $a_{k} \geq 0$ and

$$
a_{k}^{2} \leq a_{k-1} a_{k+1} \quad \text { for } \quad k \geq 1 .
$$

Log-convex functions and sequences are often used to study densities and discrete distributions in probability.

If a $\log$-convex sequence starts from $a_{0}=0$, then all further elements are also zeros, i.e., $A=0$. Another trivial case is $a_{0}=1$ and $a_{1}=0$, which gives $a_{k}=0$ for $k \geq 2$, i.e., $A=I$. Excluding these possibilities, we assume that $a_{0}=1$ and $a_{1}>0$ and claim from (2.1) that $a_{k}>0$ for $k \geq 2$, i.e., all elements of the sequence are strictly positive. This allows us to rewrite $(2.1)$ as

$$
\frac{a_{k+1}}{a_{k}} \geq \frac{a_{k}}{a_{k-1}} \quad \text { for } \quad k \geq 1 .
$$

The following theorem was known to Hardy [14, Theorem 22] and is used for error analysis of discretization methods for Volterra equations [17, Chapter 10].

TheOrem 2.2 (see [14]). If the coefficients $\left\{a_{k}\right\}$ of the triangular Toeplitz matrix are log-convex and decay slowly, then its inverse $B=A^{-1}$ is bounded, and $\|B\|_{1}=$ $\sum_{k=0}^{\infty}\left|b_{k}\right|=2$.

The proof consists of several steps. First, it is easy to show that a log-convex sequence is nonincreasing.

Lemma 2.3 (see [14]). If $\left\{a_{k}\right\}$ is log-convex and bounded, then $a_{k} \geq a_{k+1}$ for $k \geq 0$.

Proof. The statement of the lemma holds for the trivial cases, $A=0$ and $A=I$. For other sequences we see from $\left(2.1^{\prime}\right)$ that $a_{k+1} / a_{k}$ is a nondecreasing sequence, and therefore it has a limit. Since $\left\{a_{k}\right\}$ is bounded, $\lim _{k \rightarrow \infty} a_{k+1} / a_{k} \leq 1$, and $a_{k+1} / a_{k} \leq 1$ for $k \geq 0$, which completes the proof.

The central component of the proof is the following result.

Lemma 2.4 (see [14]). For a triangular Toeplitz matrix A defined by a log-convex sequence $\left\{a_{k}\right\}$ with $a_{0}=1$, the inverse matrix $B=A^{-1}$ has nonpositive elements $b_{k} \leq 0$ for all $k \geq 1$.

The proof relies significantly on the log-convexity of a sequence. We provide the proof in Appendix A, together with the rest of the proof of Theorem 2.2. If the inequality (2.1) does not hold at some point $k^{\prime}$, we cannot predict the signs of $b_{k}$ 's for $k>k^{\prime}$, and therefore cannot estimate the norm $\|B\|_{1}$ using Theorem 2.2. Particular care is required to ensure that the log-convexity is preserved during the discretization 

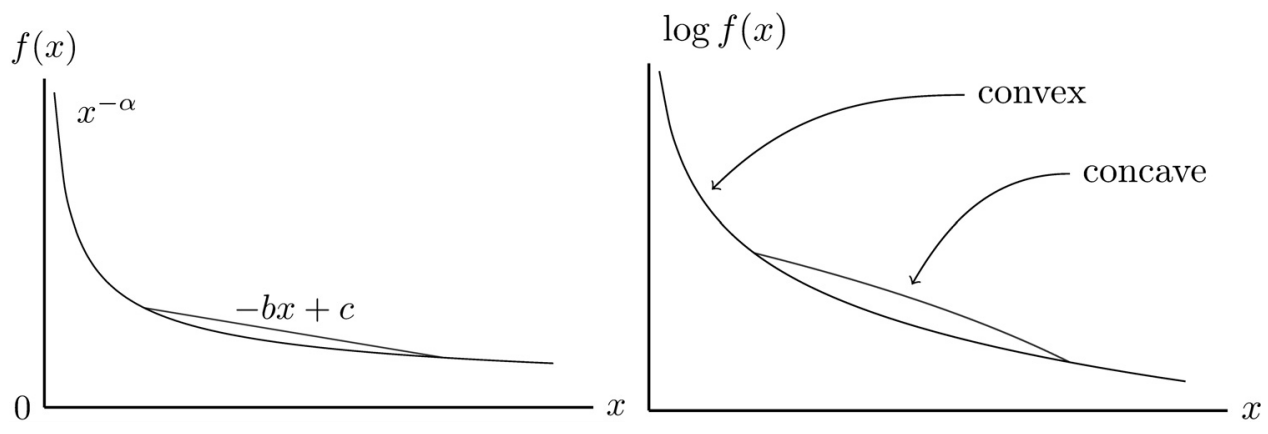

FIG. 1. Log-convexity is very delicate-a piecewise-linear approximation of the log-convex function $x^{-\alpha}$ is not a log-convex function.
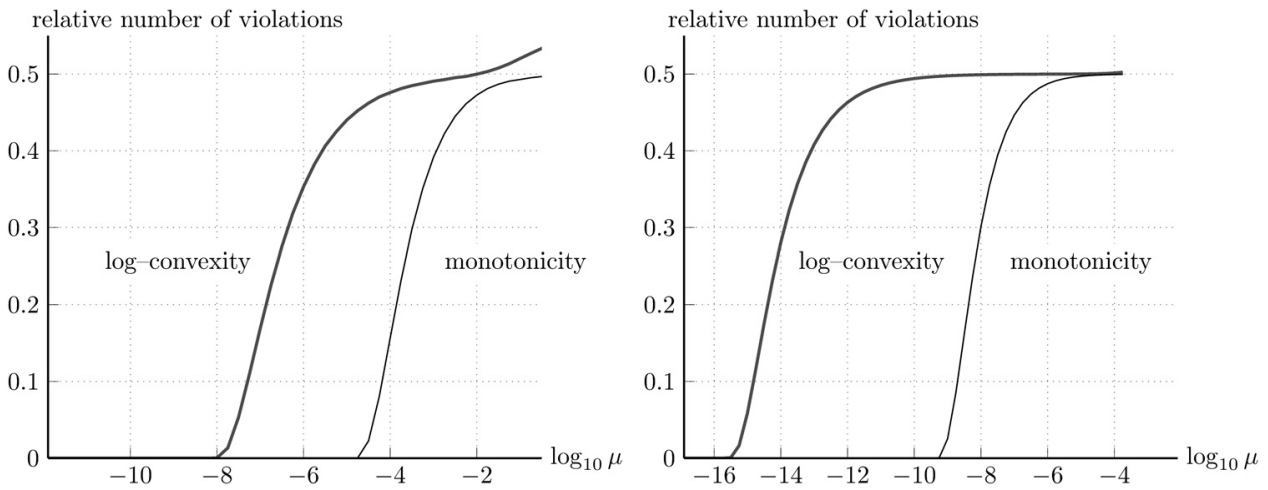

FIG. 2. Relative number of violations of monotonicity and log-convexity for a sequence $a_{k}=$ $(k+1)^{-0.5}+\mu s_{k}$, where $s_{k}$ is uniformly distributed in $[0.1)$, and $k=0, \ldots, n-1$, with sequence size $n=2^{10}$ (left) and $n=2^{20}$ (right).

and not distorted by approximation errors and noise. This is, however, a nontrivial task, as we illustrate by the following examples.

Example 2.5. The simplest way to violate the log-convexity of a function is to approximate a piece of it by a linear function, as we show in Figure 1. For example, the function $t^{-\alpha}$ and the corresponding sequence $(1+k)^{-\alpha}$ are log-convex for $\alpha>0$, because $k^{2} \geq(k-1)(k+1)$ for $k \geq 1$. However, its linear approximation of a form $-b x+c$ with some $b>0, c>0$, is not log-convex (see Figure 1, right). The corresponding sequence $-b k+c$ also does not satisfy $(2.1)$, since $(-b k+c)^{2}>$ $(-b k+c-b)(-b k+c+b)$.

Example 2.6. During the discretization, perturbations occurring due to the fixed machine precision threshold, and other sources of noise, can also violate the logconvexity of entries $\left\{a_{k}\right\}$. For example, we consider $a_{k}=(1+k)^{-\alpha}+\mu s_{k}$, where $\mu$ is the amplitude of noise and $s_{k}$ are (pseudo)random values, uniformly distributed in $[0: 1)$. For $\mu=0$ this is a perfectly ordered and log-convex sequence, but when the noise increases, first the log-convexity and then the monotonicity are violated, as we show in Figure 2. For a relatively small sequence of size $n=2^{10}$ the log-convexity is first violated for $\mu=10^{-8}$ and the monotonicity for $\mu=2 \cdot 10^{-5}$. For a larger sequence of size $n=2^{20}$ the log-convexity is violated almost instantly by a noise of amplitude $\mu=3 \cdot 10^{-16}$, which is very close to the double precision machine threshold level. The monotonicity persists until $\mu=7 \cdot 10^{-10}$. 
The example considered shows that in practical computations with large matrices we can hardly expect that the log-convexity condition (2.1) would hold for all elements of the matrix $A$. Therefore, we cannot rely on Theorem 2.2 to analyze the inverse matrix $B$. A similar problem appears if we want to apply some numerical approximations to $A$ in order to reduce the number of parameters and speed up the computations, as we do in [23]. In the rest of the paper we will try to see how much we can say about the properties of $B$ without the log-convexity of the elements of $A$.

3. The decay of the elements of the fundamental matrix. For a triangular Toeplitz matrix $A$ defined by (1.1), the fundamental matrix is defined as follows.

DeFINITION 3.1 (see [25]). The fundamental matrix $\left\{u_{k}\right\}$ is a sequence with $u_{-1}=0, u_{k}=\sum_{j=0}^{k} b_{j}$ for $k \geq 0$, where $\left\{b_{j}\right\}$ are the entries of the inverse matrix (1.2).

Remark 3.2. In this paper we do not associate $\left\{u_{k}\right\}$ with a particular matrix but preserve the name from [25] for compatibility with the existing literature.

Since $b_{k}=u_{k}-u_{k-1}, k \geq 0$, properties of the fundamental matrix, such as limit and summability, allow us to study the inverse matrix. The following elementary statements can be found in, e.g., [25].

LEMma 3.3. In the definitions made above we have

$$
\begin{array}{cc}
\sum_{j=0}^{k} a_{j} b_{k-j}=\sum_{j=0}^{k} a_{k-j} b_{j}=0 & \text { for } \quad k \geq 1 ; \\
\sum_{j=0}^{k} a_{j} u_{k-j}=\sum_{j=0}^{k} a_{k-j} u_{j}=1 \quad \text { for } \quad k \geq 0 ; \\
u_{k}=\sum_{j=0}^{k-1} u_{j} d_{k-j}=\sum_{j=1}^{k} u_{k-j} d_{j} \quad \text { for } \quad k \geq 1,
\end{array}
$$

where $d_{k}=a_{k-1}-a_{k}$ for $k \geq 1$.

Proof. Consider the nondiagonal entries of $A B=I$ to prove (3.1a). Summation over the $k^{\prime}$ leading rows of this linear system gives (3.1b) as follows:

$$
1=\sum_{k=0}^{k^{\prime}} \sum_{j=0}^{k} a_{j} b_{k-j}=\sum_{k=0}^{k^{\prime}} \sum_{j=0}^{k^{\prime}} a_{j} b_{k-j}=\sum_{j=0}^{k^{\prime}} a_{j} \sum_{k=0}^{k^{\prime}} b_{k-j}=\sum_{j=0}^{k^{\prime}} a_{j} u_{k^{\prime}-j},
$$

where we set $b_{k}=0$ for $k<0$. From (3.1b) the recurrence relation (3.1c) is written as

$$
a_{0} u_{k}=1-\sum_{j=0}^{k-1} a_{k-j} u_{j}=\sum_{j=0}^{k-1} a_{k-1-j} u_{j}-\sum_{j=0}^{k-1} a_{k-j} u_{j}=\sum_{j=0}^{k-1} d_{k-j} u_{j},
$$

which completes the proof.

If we additionally assume the monotone decay of $\left\{a_{k}\right\}$, the following nice statement can be derived.

Lemma 3.4 (see [25]). If $a_{k} \geq 0$ and $d_{k}=a_{k-1}-a_{k} \geq 0$ for all $k \geq 1$, then

$$
0 \leq u_{k} \leq 1 \quad \text { for } \quad k \geq 0
$$

Proof. The statement can be proved by the following inductive argument. Since $u_{0}=b_{0}=1$, the base of induction holds. Then, if $0 \leq u_{j} \leq 1$ for $1 \leq j \leq k-1$, we use (3.1c) to establish 


$$
\begin{aligned}
& u_{k}=\sum_{j=1}^{k} u_{k-j} d_{j} \geq 0, \\
& u_{k}=\sum_{j=1}^{k} u_{k-j} d_{j} \leq \sum_{j=1}^{k} d_{j}=1-a_{k} \leq 1,
\end{aligned}
$$

which completes the proof.

Now we show that for a triangular Toeplitz matrix $A$ with monotone slow decay the sequence of elements of the inverse matrix $B=A^{-1}$ also decays to zero.

THEOREM 3.5. If $1=a_{0}>a_{1} \geq a_{2} \geq \cdots \geq a_{k-1} \geq a_{k} \geq \cdots$ and $a_{k} \rightarrow 0$, but the series $\sum_{k=0}^{\infty} a_{k}$ diverges, then $u_{k} \rightarrow 0$.

Proof. Consider all convergent subsequences $\left\{u_{k_{t}}\right\}$ of the sequence $\left\{u_{k}\right\}$ and let

$$
u_{\star}=\max _{\left\{u_{k_{t}}\right\}} \lim _{t \rightarrow \infty} u_{k_{t}} .
$$

By $(3.2), 0 \leq u_{\star} \leq 1$. Suppose that $u_{\star}>0$. Since $\sum_{k=0}^{\infty} a_{k}$ is divergent, we can choose $N$ such that

$$
\sum_{k=0}^{N} a_{k}>\frac{2}{u_{\star}}
$$

and arbitrary small $\varepsilon$ that satisfies

$$
\varepsilon<\frac{c-1}{c^{N}-1} \frac{u_{\star}}{2}, \quad \text { where } \quad c=\frac{1}{1-a_{1}} .
$$

Denote by $\left\{j_{t}\right\}_{t=0}^{\infty}$ the subsequence of indices for which $u_{j_{t}}>u_{\star}-\varepsilon$. If the step sizes of $\left\{j_{t}\right\}$ are bounded (see Figure 3, left), i.e.,

$$
\exists h \forall t \geq 0: \quad j_{t+1}-j_{t} \leq h,
$$

then for some sufficiently large $T$ the following inequality holds:

$$
\begin{aligned}
\sum_{j=0}^{j_{T}} a_{j_{T}-j} u_{j} & \geq \sum_{t=0}^{T} a_{j_{T}-j_{t}} u_{j_{t}} \geq\left(u_{\star}-\varepsilon\right) \sum_{t=0}^{T} a_{j_{T}-j_{t}} \geq\left(u_{\star}-\varepsilon\right) \sum_{t=0}^{T} a_{h t} \\
& \geq \frac{u_{\star}-\varepsilon}{h} \sum_{t=0}^{T} a_{t}>1 .
\end{aligned}
$$

The contradiction with (3.1b) shows that the step sizes of $\left\{j_{t}\right\}$ are not bounded.

Choose $M$ such that $a_{M} \leq \varepsilon$. Since $\left\{j_{t+1}-j_{t}\right\}$ is unbounded, we can choose $T$ such that $j_{T}-j_{T-1} \geq M+N$ (see Figure 3, right). For $j_{T-1}<j<j_{T}$ all elements $u_{j}<u_{\star}-\varepsilon$, since none of them belongs to $\left\{u_{j_{t}}\right\}$. Apply (3.1c) with $k=j_{T}$ to establish

$$
\begin{aligned}
u_{\star}-\varepsilon \leq u_{k} & =d_{1} u_{k-1}+\sum_{j=2}^{M-1} d_{j} u_{k-j}+\sum_{j=M}^{k} d_{j} u_{k-j} \\
& \leq d_{1} u_{k-1}+\sum_{j=2}^{M-1} d_{j}\left(u_{\star}-\varepsilon\right)+\sum_{j=M}^{k} d_{j} \\
& \leq\left(1-a_{1}\right) u_{k-1}+a_{1}\left(u_{\star}-\varepsilon\right)+\varepsilon .
\end{aligned}
$$




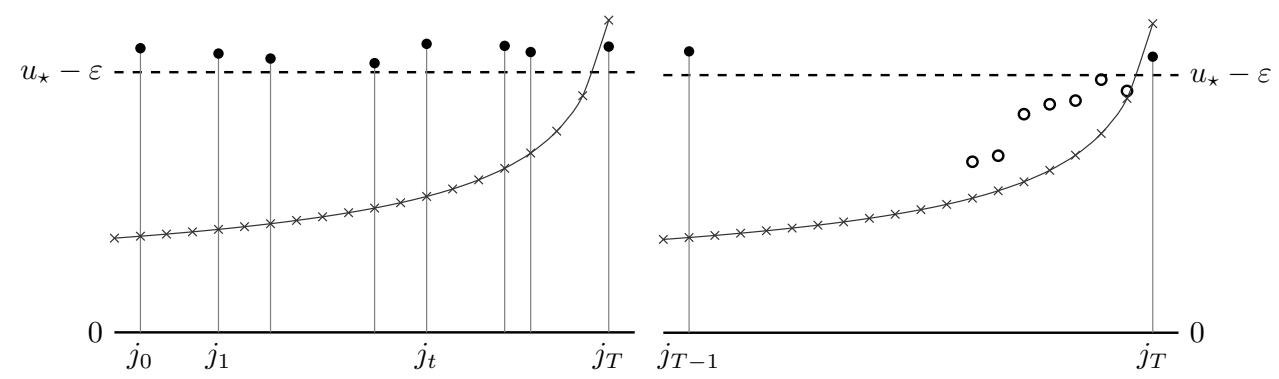

FIG. 3. Illustration of the proof of Theorem 3.5. $\circ=$ sequence $u_{j}, \bullet=$ subsequence $u_{j_{t}}$, $\times=$ sequence $a_{j_{T}-j}$.

This shows that

$$
u_{k-1} \geq u_{\star}-\left(1+\frac{1}{1-a_{1}}\right) \varepsilon=u_{\star}-c_{1} \varepsilon, \quad c_{1}=1+c=\frac{c^{2}-1}{c-1} .
$$

We proceed with induction, assuming that for some $j<N$ the inequality $u_{k-j+1} \geq$ $u_{\star}-c_{j-1} \varepsilon$ holds with $c_{j-1}=\frac{c^{j}-1}{c-1}$. Similarly to (3.3) we establish

$$
u_{k-j} \geq u_{\star}-\left(1+\frac{c_{j-1}}{1-a_{1}}\right) \varepsilon=u_{\star}-c_{j} \varepsilon, \quad c_{j}=1+\frac{c^{j}-1}{c-1} c=\frac{c^{j+1}-1}{c-1} .
$$

Using the assumption on $\varepsilon$ we conclude that

$$
u_{k-j} \geq u_{\star}-c_{j} \varepsilon>\frac{u_{\star}}{2} \quad \text { for } \quad j=0, \ldots, N-1 .
$$

Now we are ready to show the contradiction with (3.1b). Indeed, for $k=j_{T}$,

$$
\sum_{j=0}^{k} a_{j} u_{k-j} \geq \sum_{j=0}^{N} a_{j} u_{k-j}>\frac{u_{\star}}{2} \sum_{j=0}^{N} a_{j}>1
$$

The contradiction with (3.1b) proves $u_{\star}=0$, and therefore $\exists \lim _{k \rightarrow \infty} u_{k}=0$.

Corollary 3.6. Under the conditions of Theorem 3.5, $\exists \lim _{k \rightarrow \infty} b_{k}=0$.

Remark 3.7. The requirement $a_{1}<1$ in Theorem 3.5 can be removed.

Proof. Consider the minimal index $l$ such that $a_{l}<1$. Define $N$ such that $\sum_{k=0}^{N} a_{l k}>2 / u_{\star}, c=1 /\left(1-a_{l}\right)$, and $\varepsilon$ and $M$ in the same way as in the proof of the theorem. Choose $T$ such that $j_{T}-j_{T-1}>M+l N$, set $k=j_{T}$, and substitute (3.3) by

$$
u_{\star}-\varepsilon \leq u_{k}=d_{l} u_{k-l}+\sum_{j=l+1}^{M-1} d_{j} u_{k-j}+\sum_{j=M}^{k} d_{j} u_{k-j} \leq\left(1-a_{l}\right) u_{k-l}+a_{l}\left(u_{\star}-\varepsilon\right)+\varepsilon .
$$

This gives $u_{k-l}>u_{\star}-c_{1} \varepsilon$ and $u_{k-j l}>u_{\star}-c_{j} \varepsilon$ for $j=0, \ldots, N-1$. We have the same contradiction as in the proof of Theorem 3.5.

4. Summability of the fundamental matrix in $\boldsymbol{p}$-norms. In [26] it is shown that under the conditions of Theorem 3.5 the series of the fundamental matrix $\sum_{k=0}^{\infty} u_{k}$ is not convergent. The result of Theorem 3.5 shows $u_{k} \rightarrow 0, \sum_{k=0}^{\infty} u_{k}=\infty$, i.e., the sequence $u=\left\{u_{k}\right\}$ has slow decay. 
Given a sequence $a=\left\{a_{k}\right\}_{k=0}^{\infty}$ with slow decay, we sometimes can choose a power $p$ such that $\sum_{k=0}^{\infty}\left|a_{k}\right|^{p}<\infty$. A notable example is the harmonic series $\sum_{k=1}^{\infty} 1 / k$ which is divergent, but the overharmonic series $\sum_{k=1}^{\infty} 1 / k^{p}$ converges for any $p>1$.

A quantitative measure of divergence for a sequence can be given in terms of its summability in $p$-norm.

Definition 4.1. For a sequence $a=\left\{a_{k}\right\}_{k=0}^{\infty}$ we define $\|a\|_{p}$ by

$$
\|a\|_{p}^{p}=\sum_{k=0}^{\infty}\left|a_{k}\right|^{p}, \quad\|a\|_{\infty}=\sup _{k}\left|a_{k}\right|
$$

The space of sequences a with $\|a\|_{p}<\infty$ is denoted by $\ell^{p}$.

Remark 4.2. For $p \geq 1$ the operation $\|\cdot\|_{p}$ is a norm on $\ell^{p}$.

LEMma 4.3. The function $\|\cdot\|_{p}$ is decreasing in $p$ for $p>0$.

Proof. We have to show that for $0<p \leq q$ and any sequence $a$ it holds that $\|a\|_{p} \geq$ $\|a\|_{q}$. For $a=0$ this is obviously true. For the nontrivial case $\|a\|_{q} \geq \sup _{k}\left|a_{k}\right|>0$ and we can consider $x=\left\{x_{k}\right\}_{k=0}^{\infty}$ with $x_{k}=a_{k} /\|a\|_{q}$. Since $x_{k} \leq 1$ for all $k \geq 0$ it holds $x_{k}^{p} \geq x_{k}^{q}$ and hence $\|x\|_{p}^{p} \geq\|x\|_{q}^{q}$. Since $\|x\|_{q}=1$ this implies $\|x\|_{p} \geq 1$ and consequently $\|a\|_{p} \geq\|a\|_{q}$, which completes the proof.

Corollary 4.4. For $0<p \leq q$ the embedding $\ell^{q} \subseteq \ell^{p}$ holds.

Considering the above, for the sequences with slow decay the following definition makes sense.

Definition 4.5. For a sequence $a=\left\{a_{k}\right\}_{k=0}^{\infty}$ with $a_{k} \rightarrow 0$ and $\sum_{k=0}^{\infty}\left|a_{k}\right|=\infty$, find $p \geq 1$ such that $a \notin \ell^{p}$ but $a \in \ell^{q}$ for all $q>p$. The value $0 \leq 1 / p \leq 1$ will be referred to as the decay rate of $a$.

Example 4.6. The harmonic sequence $a_{k}=(k+1)^{-1}$ has decay rate 1 .

Example 4.7. The subharmonic sequence $a_{k}=(1+k)^{-\alpha}, 0<\alpha \leq 1$, has decay rate $\alpha$.

Remark 4.8. The reverse of the last example is not true: if a sequence has decay rate $\alpha$, we cannot claim that $a_{k} \leq c(1+k)^{-\alpha}$ with some $c>1$.

The analysis of the decay rate of the fundamental matrix is based on Young's convolution theorem [28]. It is one of the most basic results in harmonic analysis, which plays an important role, e.g., in PDE theory.

THEOREM 4.9 (Young's inequality for discrete convolution). Let $z_{k}=$ $\sum_{j=0}^{k} x_{j} y_{k-j}$ for $k \geq 0$. For $1 \leq p, q, r \leq \infty$ such that

$$
1+\frac{1}{r}=\frac{1}{p}+\frac{1}{q}
$$

it follows that

$$
\|z\|_{r} \leq\|x\|_{p}\|y\|_{q} .
$$

The discrete version of this theorem is not common in the literature and we provide the proof in Appendix B. Using this inequality, we can estimate the decay rate of the fundamental matrix.

THEOREM 4.10. Consider a triangular Toeplitz matrix generated by a nonnegative slowly decaying sequence

$$
a=\left\{a_{k}\right\}_{k=0}^{\infty}, \quad a_{k} \geq 0, \quad \lim _{k \rightarrow \infty} a_{k}=0, \quad \sum_{k=0}^{\infty} a_{k}=\infty .
$$

If a has decay rate $0 \leq \alpha \leq 1$, the fundamental matrix has decay rate $v \leq 1-\alpha$. 

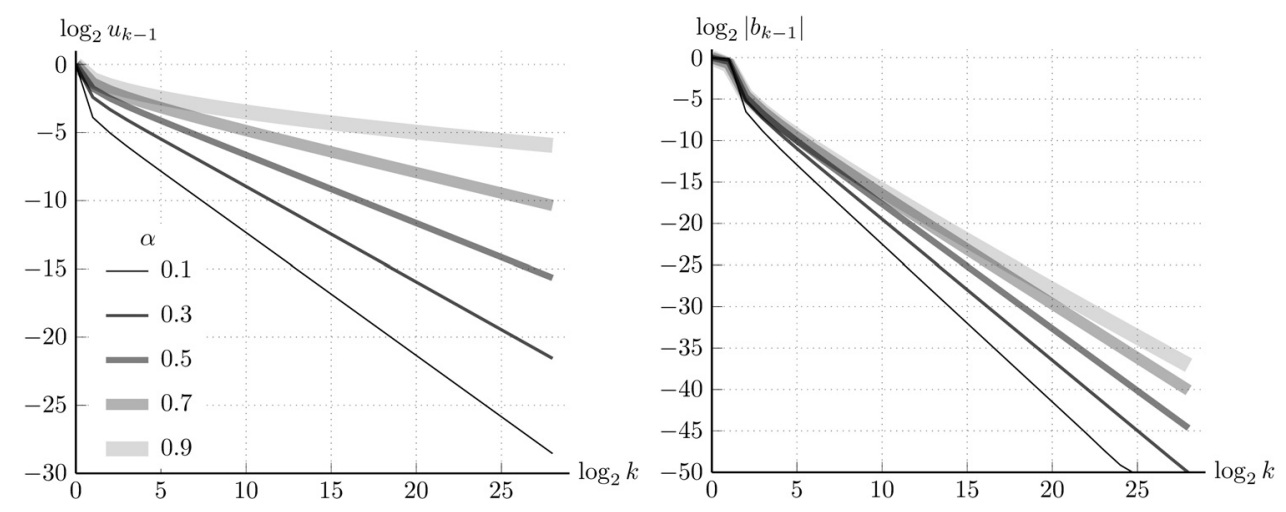

FIG. 4. Decay of the fundamental matrix (left) and the inverse matrix (right) for the triangular Toeplitz matrix with the elements $a_{k}=(1+k)^{-\alpha}, k \geq 0$, for different $\alpha<1$.

Proof. The result of Vecchio [26] proves $v<1$. Suppose that $\alpha+v>1$; then according to Definition 4.5

$$
\exists p, q \geq 1, \quad \frac{1}{p}+\frac{1}{q}>1, \quad \text { such that } \quad\|a\|_{p}<\infty, \quad\|u\|_{q}<\infty .
$$

By Young's inequality for the sequence $z_{k}=\sum_{j=0}^{k} a_{j} c_{k-j}$ there is $1<r<\infty$ such that

$$
\|z\|_{r} \leq\|a\|_{p}\|c\|_{q}<\infty .
$$

However, by (3.1b), $z_{k}=1$ for all $k \geq 0$ and $\|z\|_{r}=\infty$ for all $r<\infty$. The conclusion of the theorem follows by contradiction.

5. Numerical examples. First, we consider the sequence $a_{k}=(1+k)^{-\alpha}, k \leq 0$, that is $\log$-convex and has slow decay for $\alpha<1$, and the corresponding triangular Toeplitz matrix $A$ defined by (1.1). For different values of $\alpha$ we have computed the inverse matrix $B=A^{-1}$ using the divide-and-conquer algorithm [21, 9]. We show the behavior of the elements of the inverse and the fundamental matrix in Figure 4. We observe that the rate of decay $v$ for the elements $u_{k}$ of the fundamental matrix behaves as prescribed by Theorem 4.10, i.e., $v=1-\alpha$. Note that the example considered seems to provide the sharp bound for the inequality in Theorem 4.10, but we do not have a theoretical proof of this fact yet. Since the sequence $a_{k}$ is log-convex, we have by Lemma 2.4 that $b_{k} \leq 0$ for $k \geq 1$, and therefore the sequence of elements of the fundamental matrix $u_{k}$ is nonincreasing by Theorem 2.2. It is no surprise that the elements of the inverse matrix, which are in fact the numerical derivative of $u_{k}$, have the decay rate $\beta=1+v=2-\alpha$. This behavior is clearly observed in Figure 4 .

Our second example is the sequence $\left\{a_{k}\right\}$ with $a_{0}=1$ and $a_{k}=c(1+k)^{-\alpha}$ for $k \geq 1$, where $c \leq 1$ is a variable parameter. This pattern of elements is typical for convolution Volterra equations of the second kind [3, 4, 23], in which case $c \sim h^{\alpha}$, where $h$ is the grid step. Of course, for $c=1$ we observe the same results as in Figure 4. For small $c$ the considered sequence has a certain jump at the very beginning but is still perfectly monotone and log-convex everywhere, just like in the previous example. Consequently, we observe the same asymptotic behavior for sequences $\left\{b_{k}\right\}$ and $\left\{u_{k}\right\}$ at the limit, but in this case it does not start instantly, as we see in Figure 5. 

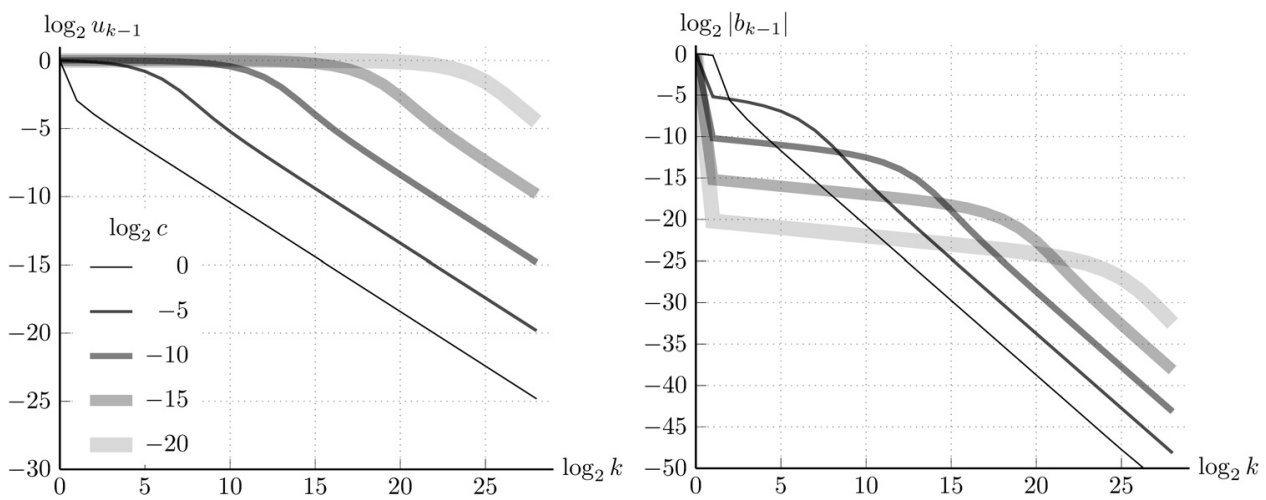

FIG. 5. Decay of the fundamental matrix (left) and the inverse matrix (right) for the triangular Toeplitz matrix with the elements $a_{0}=1$ and $a_{k}=c(1+k)^{-\alpha}, k \leq 1$, for $\alpha=0.2$ and different $c \leq 1$.

Instead, the sequence $\left\{u_{k}\right\}$ stays around 1 for quite some time, while $\left\{b_{k}\right\}$ behaves approximately as $b_{k} \simeq-a_{k}=-c(1+k)^{-\alpha}$ for $k \lesssim k_{\star}$. For $k \gtrsim k_{\star}$, both $\left\{u_{k}\right\}$ and $\left\{b_{k}\right\}$ switch to the expected decay rates $v=1-\alpha$ and $\beta=2-\alpha$, respectively. Due to the log-convexity of $a_{k}$ we know from Theorem 2.2 that $b_{k} \leq 0$ for $k \geq 1$, and therefore $u_{k}$ is nonincreasing. This allows us to estimate the position of the critical point $k_{\star}$ between two régimes from the condition $1+\sum_{k=1}^{k_{\star}} b_{k} \simeq 0$, which gives $c k_{\star}^{1-\alpha} /(1-\alpha) \simeq 1$, and $k_{\star} \simeq c^{-\frac{1}{1-\alpha}}$. The data in Figure 5 agree with this estimate. This example illustrates why it can be difficult to provide a meaningful estimate for $\left|b_{k}\right|$ using only the asymptotic properties of $\left\{a_{k}\right\}$ (such as decay rate), unless $k$ is very large.

Finally, we consider the sequence $\left\{a_{k}\right\}$ with $a_{0}=a_{1}=1$ and $a_{k}=c(1+k)^{-\alpha}$ for $k \geq 2$, where $c \leq 1$ is again a variable parameter. The log-convexity is violated in this sequence in just one point $k=1$, where $1=a_{1}^{2} \geq a_{0} a_{2}=3^{-\alpha}$. The monotonicity is preserved everywhere, and the decay rate is equal to $\alpha$, like in the previous examples. For small $c$ we can consider this sequence as an approximation of the bidiagonal matrix in (1.4), and therefore the elements of the inverse $B=A^{-1}$ are expected to behave similarly to the sign-alternating sequence in (1.4). Therefore, in the beginning of the sequence $\left\{b_{k}\right\}$ all even entries are close to 1 , and odd entries are close to -1 . Therefore, the entries of $\left\{b_{k}\right\}$ alternate signs, but all $\left|b_{k}\right|$ 's remain close to 1 . In contrast, the sequence $\left\{u_{k}\right\}$ is nonnegative but "highly oscillatory" in the beginning, i.e., $u_{-1}=0, u_{0}=1, u_{1}=0, u_{2} \approx 1$, and so on. To demonstrate the decay pattern of the fundamental matrix, ignoring the fast oscillations, we show in Figure 6 the odd and even entries of $\left\{u_{k}\right\}$ separately. We can see that after a certain critical point $k_{\star}$ the values of $\left|b_{k}\right|$ tend toward zero with the decay rate $\beta=2-\alpha$, while $\left|u_{k}\right|$ decays with the rate $v=1-\alpha$, just as in the previous examples. The critical point $k_{\star}$ seems to be at the same position $k_{\star} \simeq c^{-\frac{1}{1-\alpha}}$ as the one in Figure 5 . It is clear from this example that for every $0<\alpha<1$ we can choose a sufficiently small $c$ such that the asymptotic decay of $\left|b_{k}\right|$ starts only after a very large $k_{\star}$ and the norm $\|B\|_{1}=\sum_{k=0}^{\infty}\left|b_{k}\right|$ is arbitrarily large. Therefore, if the log-convexity (2.1) of $A$ is violated at just one point, there is no hope to construct a uniform upper bound for the inverse.

6. Conclusion. Classical methods for the error analysis of Volterra equations of the first kind are based on the log-convexity of the elements of triangular Toeplitz 

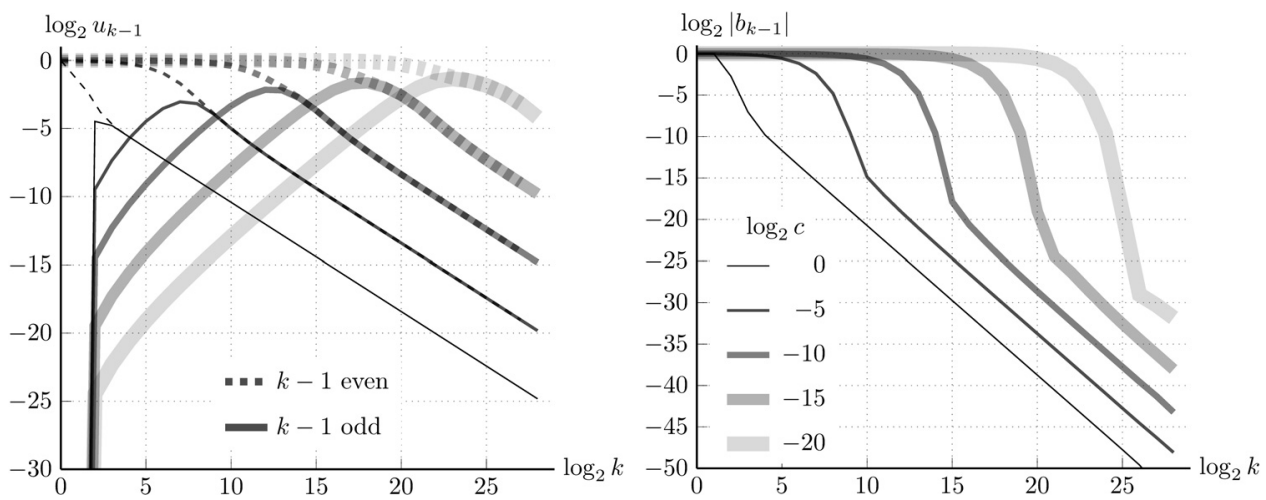

FIG. 6. Decay of the fundamental matrix (left) and the inverse matrix (right) for the triangular Toeplitz matrix with the elements $a_{0}=a_{1}=1$ and $a_{k}=c(1+k)^{-\alpha}, k \geq 2$, for $\alpha=0.2$ and different $c \leq 1$.

matrices of the corresponding linear systems; see Theorem 2.2. However, this property is very delicate and can be easily violated by approximations, perturbations, or noise. In this paper we consider matrices with slow decay of the elements and establish new results on the decay of the inverse and the fundamental matrix that are not based on the log-convexity. In particular, we show that the elements of the fundamental matrix $\left\{u_{k}\right\}$ and the inverse matrix $\left\{b_{k}\right\}$ decay to zero (see Theorem 3.5) and provide a quantitative description of this decay in Theorem 4.10. By numerical examples we demonstrate that the decay rate prescribed by our analysis manifests itself for the "tails" of $\left\{u_{k}\right\}$ and $\left\{b_{k}\right\}, k>k_{\star}$, beyond some critical point $k_{\star}$, which can be large. We also show that if the log-convexity is violated in just a single point, the norm of the inverse matrix can be arbitrary large. The proposed results add to the classical analysis of Jaffard [15] and to the results of Vecchio and others [25, 26, 2]. The predicted decay rates together with the empirical estimate of $k_{\star}$ can be used to develop fast algorithms for the solution of convolution Volterra equations in the spirit of [23].

Appendix A. Theorems concerning the log-convex case. We consider a $\log$-convex sequence (2.1) and provide proofs for the lemma and the theorem in section 2. Since $A$ is inverible, we will assume w.l.o.g. $a_{0}=1$.

If $a_{1}=0$ and $a$ is $\log$-convex, then all further elements of the sequence are zeroes. Indeed, $a_{2}^{2} \leq a_{1} a_{3}=0$ hence $a_{2}=0$ and so on. This sequence defines the unit matrix $A=I$ with $B=A^{-1}=I$, for which the result of Lemma 2.4 holds.

If $a_{1}>0$, then all further elements of the log-convex sequence are also strictly positive - to prove this, apply $a_{k+1} \geq a_{k}^{2} / a_{k-1}>0$ recursively for $k \geq 2$. Therefore all elements of $a$ are nonzeroes and we can divide by them, obtaining $\left(2.1^{\prime}\right)$.

Proof of Lemma 2.4. Since $a_{0}=1,(1.2)$ gives $b_{1}=-a_{1} \leq 0$, which is the base of induction. Assume that $b_{j} \leq 0$ for $j=1, \ldots, k$ and prove the same for $b_{k+1}$. From (3.1a) it follows that $a_{k}=-\sum_{j=1}^{k} a_{k-j} b_{j}$ and for $k \geq 1$ we have

$$
\begin{aligned}
& -\frac{a_{k}}{a_{k-1}}=\sum_{j=1}^{k} \frac{a_{k-j}}{a_{k-1}} b_{j}, \\
& -\frac{a_{k+1}}{a_{k}}=\sum_{j=1}^{k+1} \frac{a_{k+1-j}}{a_{k}} b_{j}=\sum_{j=1}^{k} \frac{a_{k+1-j}}{a_{k}} b_{j}+\frac{b_{k+1}}{a_{k}} .
\end{aligned}
$$


Subtracting one line from another, we obtain

$$
\frac{a_{k}}{a_{k-1}}-\frac{a_{k+1}}{a_{k}}=\sum_{j=1}^{k}\left(\frac{a_{k+1-j}}{a_{k}}-\frac{a_{k-j}}{a_{k-1}}\right) b_{j}+\frac{b_{k+1}}{a_{k}},
$$

where the left-hand side is nonpositive since $a$ is log-convex. Similarly, all parentheses in the right-hand side are nonpositive due to the $\log$-convexity of $a$,

$$
\begin{aligned}
\frac{a_{k+1-j}}{a_{k-j}}-\frac{a_{k}}{a_{k-1}}= & \left(\frac{a_{k+1-j}}{a_{k-j}}-\frac{a_{k+2-j}}{a_{k+1-j}}\right)+\left(\frac{a_{k+2-j}}{a_{k+1-j}}-\frac{a_{k+3-j}}{a_{k+2-j}}\right) \\
& +\cdots+\left(\frac{a_{k-1}}{a_{k-2}}-\frac{a_{k}}{a_{k-1}}\right) \leq 0 .
\end{aligned}
$$

We conclude that

$$
\frac{b_{k+1}}{a_{k}}=\left(\frac{a_{k}}{a_{k-1}}-\frac{a_{k+1}}{a_{k}}\right)-\sum_{j=1}^{k}\left(\frac{a_{k+1-j}}{a_{k}}-\frac{a_{k-j}}{a_{k-1}}\right) b_{j} \leq 0 .
$$

Each set of parentheses in the right-hand side is nonpositive, and all $b_{j} \leq 0, j=$ $1, \ldots, k$, are nonpositive by the inductive assumption. It follows that $b_{k+1} \leq 0$, and the theorem is proved by induction.

To prove Theorem. 2.2, we combine the result of Lemma 2.4 with Theorem 3.5. It follows that $1+\sum_{k=1}^{\infty} b_{k}=1-\sum_{k=1}^{\infty}\left|b_{k}\right|=0$ and $\|B\|_{1}=1+\sum_{k=1}^{\infty}\left|b_{k}\right|=1+1=2$.

Appendix B. Young's inequality for discrete convolutions. Here we provide the proof of Young's convolution theorem, Theorem 4.9, for sequences. We start with several lemmas.

Lemma B.1 (Young's inequality for products [27]). For real numbers $x, y \geq 0$ and $p, q \geq 1$ such that $1 / p+1 / q=1$, it holds that

$$
x y \leq \frac{x^{p}}{p}+\frac{y^{q}}{q} .
$$

Proof. Since the logarithmic function is concave, it holds that

$$
\log (x y)=\log x+\log y=\frac{1}{p} \log x^{p}+\frac{1}{q} \log y^{q} \leq \log \left(\frac{x^{p}}{p}+\frac{y^{q}}{q}\right),
$$

and exponentiation completes the proof.

Lemma B.2 (Hölder's inequality). For sequences $x \in \ell^{p}$ and $y \in \ell^{q}$ with $p, q \geq 1$ and $1 / p+1 / q=1$, it holds that

$$
\|x y\|_{1} \leq\|x\|_{p}\|y\|_{q}
$$

where $z=x y$ denotes the elementwise product of sequences, i.e., $z_{j}=x_{j} y_{j}, j \geq 0$.

Proof. If $\|x\|_{p}=0$ or $\|y\|_{q}=0$, then $x y=0$ and the result is trivial. For nonzero $x$ and $y$ w.l.o.g. we set $\|x\|_{p}=\|y\|_{q}=1$. Then using (B.1) we write

$$
\sum_{j=0}^{\infty}\left|x_{j} y_{j}\right| \leq \sum_{j=0}^{\infty}\left(\frac{\left|x_{j}\right|^{p}}{p}+\frac{\left|y_{j}\right|^{q}}{q}\right) \leq \frac{\|x\|_{p}^{p}}{p}+\frac{\|y\|_{q}^{q}}{q}=\frac{1}{p}+\frac{1}{q}=1
$$

which completes the proof. 
Lemma B.3 (generalized Hölder's inequality). If $\sum_{k=1}^{m} 1 / p_{k}=1 / r$ for $p_{k}>0$ and $0<r<\infty$, and sequences $x_{k} \in \ell^{p_{k}}$, then

$$
\left\|x_{1} x_{2} \cdots x_{m}\right\|_{r} \leq\left\|x_{1}\right\|_{p_{1}}\left\|x_{2}\right\|_{p_{2}} \ldots\left\|x_{m}\right\|_{p_{m}} .
$$

Proof. For $m=1$ the result is obvious, which gives us the base of induction. Suppose the result holds for $m-1$ sequences $x_{1}, \ldots, x_{m-1}$ and prove the same for $m$. If $p_{m}=\infty$, we can pull out the supremum of $\left|x_{m}\right|$ and use the induction hypothesis. For $p_{m}<\infty$ consider $1 / p=1-r / p_{m}$ and $1 / q=r / p_{m}$, which form a Hölder pair $1 / p+1 / q=1$. Since $p, q \geq 1$, we can use (B.2) to obtain

$$
\begin{aligned}
\left\|\left|x_{1} \cdots x_{m-1}\right|^{r}\left|x_{m}\right|^{r}\right\|_{1} & \leq\left\|\left|x_{1} \ldots x_{m-1}\right|^{r}\right\|_{p}\left\|\left|x_{m}\right|^{r}\right\|_{q}, \\
\left\|x_{1} \cdots x_{m-1} x_{m}\right\|_{r} & \leq\left\|x_{1} \ldots x_{m-1}\right\|_{p r}\left\|x_{m}\right\|_{q r} .
\end{aligned}
$$

Since $q r=p_{m}$ and $\sum_{k=1}^{m-1} 1 / p_{k}=1 / r-1 / p_{m}=1 /(p r)$, the lemma is proved by induction.

Now we are ready to prove the convolution inequality. The structure of the proof follows [5, Theorem 3.9.4]

Proof of Theorem 4.9. We formally extend the sequence $\left\{y_{j}\right\}_{j=0}^{\infty}$ with entries $y_{j}=0$ for $j<0$. This does not change its norm. Assuming w.l.o.g. that $p \leq q$, we start from the following simple cases:

(A) $p=q=r=1$. It is sufficient to write

$$
\|z\|_{1}=\sum_{k=0}^{\infty}\left|z_{k}\right| \leq \sum_{k=0}^{\infty} \sum_{j=0}^{k}\left|x_{j} y_{k-j}\right|=\sum_{j=0}^{\infty}\left|x_{j}\right| \sum_{k=j}^{\infty}\left|y_{k-j}\right|=\|x\|_{1}\|y\|_{1} .
$$

(B) $r=\infty, 1 / p+1 / q=1$. Since $\left|z_{k}\right| \leq \sum_{j=0}^{k}\left|x_{j} y_{k-j}\right|=\sum_{j=0}^{\infty}\left|x_{j} y_{k-j}\right|$, the result follows from Hölder's inequality (B.2) applied to sequences $\hat{x}=\left\{\left|x_{j}\right|\right\}_{j=0}^{\infty}$ and $\hat{y}=\left\{\left|y_{k-j}\right|\right\}_{j=0}^{\infty}$, taking into account that $\|\hat{x}\|_{p}=\|x\|_{p}$ and $\|\hat{y}\|_{q} \leq\|y\|_{q}$.

(C) $p=1,1<q=r<\infty$. Consider $q^{\prime}>1$ such that $1 / q+1 / q^{\prime}=1$. For any $k$, by Hölder's inequality (B.2) we have

$$
\begin{aligned}
\left|z_{k}\right| & =\left|\sum_{j=0}^{k} x_{j} y_{k-j}\right| \leq \sum_{j=0}^{k}\left|x_{j} y_{k-j}\right|=\sum_{j=0}^{\infty}\left|x_{j} y_{k-j}\right|=\sum_{j=0}^{\infty}\left(\left|y_{k-j}\right|^{1 / q^{\prime}}\right)\left(\left|x_{j}\right|\left|y_{k-j}\right|^{1 / q}\right) \\
& \leq\left(\sum_{j=0}^{\infty}\left|y_{k-j}\right|\right)^{1 / q^{\prime}}\left(\sum_{j=0}^{\infty}\left|x_{j}\right|^{q}\left|y_{k-j}\right|\right)^{1 / q} \leq\|y\|_{1}^{1 / q^{\prime}}\left(\sum_{j=0}^{\infty}\left|x_{j}\right|^{q}\left|y_{k-j}\right|\right)^{1 / q}, \\
\|z\|_{q}^{q} & =\sum_{k=0}^{\infty}\left|z_{k}\right|^{q} \leq\|y\|_{1}^{q / q^{\prime}} \sum_{k=0}^{\infty} \sum_{j=0}^{\infty}\left|x_{j}\right|^{q}\left|y_{k-j}\right|=\|y\|_{1}^{1+q / q^{\prime}}\|x\|_{q}^{q}=\|y\|_{1}^{q}\|x\|_{q}^{q},
\end{aligned}
$$

which completes the proof of case $(\mathrm{C})$.

Finally, let $1<p \leq q<r<\infty$. For each $k$ consider again the sequences $\hat{x}$ and $\hat{y}$, write

$$
\left|x_{j} y_{k-j}\right|=\left(\left|x_{j}\right|^{p}\left|y_{k-j}\right|^{q}\right)^{1 / r}\left|x_{j}\right|^{1-p / r}\left|y_{k-j}\right|^{1-q / r},
$$

and apply generalized Hölder inequality (B.3) with

$p_{1}=r, \quad p_{2}=\frac{p}{1-p / r}, \quad p_{3}=\frac{q}{1-1 / r}, \quad \frac{1}{p_{1}}+\frac{1}{p_{2}}+\frac{1}{p_{3}}=\frac{1}{r}+\frac{1}{p}-\frac{1}{r}+\frac{1}{q}-\frac{1}{r}=1$. 
We have

$$
\begin{aligned}
& \left|z_{k}\right| \leq \sum_{j=0}^{k}\left|x_{j} y_{k-j}\right| \leq\left(\sum_{j=0}^{k}\left|x_{j}\right|^{p}\left|y_{k-j}\right|^{q}\right)^{1 / r}\|x\|_{p}^{1-p / r}\|y\|_{q}^{1-q / r}, \\
& \|z\|_{r}^{r} \leq\|x\|_{p}^{r-p}\|y\|_{q}^{r-q} \sum_{k=0}^{\infty} \sum_{j=0}^{\infty}\left|x_{j}\right|^{p}\left|y_{k-j}\right|^{q}=\|x\|_{p}^{r}\|y\|_{q}^{r},
\end{aligned}
$$

which completes the proof.

\section{REFERENCES}

[1] M. Benzi And G. H. Golub, Bounds for the entries of matrix functions with applications to preconditioning, BIT, 39 (1999), pp. 417-438.

[2] K. S. Berenhaut, D. C. Morton, and P. T. Fletcher, Bounds for inverses of triangular Toeplitz matrices, SIAM J. Matrix Anal. Appl., 27 (2005), pp. 212-217.

[3] L. Blanck, Stability of collocation for weakly singular Volterra equations, IMA J. Numer. Anal., 15 (1995), pp. 357-375.

[4] L. Blanck, Stability results for collocation methods for Volterra integral equations, J. Appl. Math. Comput., 79 (1996), pp. 267-288.

[5] V. I. BogacheV, Measure Theory, Springer-Verlag, Berlin, 2007.

[6] H. Brunner, On systems of Volterra difference equations associated with collocation methods for weakly singular Volterra integral equations, in New Developments in Difference Equations and Applications, Gordon and Breach, Amsterdam, 1999, pp. 75-92.

[7] H. Brunner, Collocation Methods for Volterra Integral and Related Functional Equations, Cambridge University Press, Cambridge, UK, 2004.

[8] M. Caputo and F. Mainardi, Linear models of dissipation in anelastic solids, Riv. Nuovo Cimento, 1 (1971), pp. 161-198.

[9] D. Commenges And M. Monsion, Fast inversion of triangular Toeplitz matrices, IEEE Trans. Automat. Control, 29 (1984), pp. 250-251.

[10] S. Demko, W. F. Moss, And P. W. Smith, Decay rates for inverses of band matrices, Math. Comp., 43 (1984), pp. 491-499.

[11] K. Diethelm, An algorithm for the numerical solution of differential equations of fractional order, Electron. Trans. Numer. Anal., 5 (1997), pp. 1-6.

[12] A. Freed And K. Diethelm, Fractional calculus in biomechanics: A 3D viscoelastic model using regularized fractional-derivative kernels with application to the human calcaneal fat pad, Biomech. Model. Mechanobiol., 5 (2006), pp. 203-215.

[13] G. Gripenberg, On Volterra equations of the first kind, Integral Equations Operator Theory, 3 (1980), pp. 473-488.

[14] G. H. Hardy, Divergent Series, Clarendon Press, Oxford, UK, 1949.

[15] S. Jaffard, Propriétés des matrices «bien localisées» près de leur diagonale et quelques applications, Ann. Inst. H. Poincaré Anal. Non Linéare, 7 (1990), pp. 461-476.

[16] J. F. C. Kingman, A convexity property of positive matrices, Quart. J. Math. Oxford, 12 (1961), pp. 283-284.

[17] P. Linz, Analytical and Numerical Methods for Volterra Equations, SIAM, Philadelphia, 1985.

[18] X. Liu, S. McKee, J. Y. Yuan, and Y. X. Yuan, Uniform bounds of the 1-norm of the inverse of lower triangular Toeplitz matrices, Linear Algebra Appl., 435 (2011), pp. 1157-1170.

[19] C. Lubich, A stability analysis of convolution quadratures for Abel-Volterra integral equations, IMA J. Numer. Anal., 6 (1986), pp. 87-101.

[20] G. N. Minerbo And E. L. Maurice, Inversion of Abel's integral equations by means of orthogonal polynomials, SIAM J. Numer. Anal., 6 (1969), pp. 598-616.

[21] M. MorF, Doubling algorithms for Toeplitz and related equations, in Acoustics, Speech and Signal Processing, IEEE, 1980, pp. 954-959.

[22] O. H. Nestor And H. N. Olsen, Numerical methods for reducing line and surface probe data, SIAM Rev., 2 (1960), pp. 200-207. 
[23] J. A. Roberts, D. V. Savostyanov, and E. E. Tyrtyshnikov, Superfast solution of linear convolutional Volterra equations using QTT approximation, J. Comput. Appl. Math., 260 (2014), pp. 434-448.

[24] J. B. Tatum and W. A. Jaworski, A solution of Abel's equation, J. Quant. Spectrosc. Radiat. Transfer, 38 (1987), pp. 319-322.

[25] A. Vecchio, Volterra discrete equations: Summability of the fundamental matrix, Numer. Math., 81 (2001), pp. 783-794.

[26] A. Vecchio, A bound for the inverse of a lower triangular Toeplitz matrix, SIAM J. Matrix Anal. Appl., 24 (2003), pp. 1167-1174.

[27] W. H. Young, On classes of summable functions and their Fourier series, Proc. Roy. Soc. London Ser. A, 87 (1912), pp. 225-229.

[28] W. H. Young, On the multiplication of successions of Fourier constants, Proc. Roy. Soc. London Ser. A, 87 (1912), pp. 331-339. 